\title{
USO DE UM SCANNER DE MESA E ESPUMA DE POLIURETANO PARA QUANTIFICAÇÃO DE FERRO TOTAL EM CÁPSULAS DE SUPLEMENTOS ALIMENTARES
}

\author{
Fernanda. N. Feiteira ${ }^{a}$, João V. de P. P. Barreiros ${ }^{\mathrm{a}}$ e Wagner F. Pacheco ${ }^{\mathrm{a}, * \text {, (i) }}$ \\ aDepartamento de Químcia Analítica, Instituto de Química, Universidade Federal Fluminense, 24020-141 Niterói - RJ, Brasil
}

Recebido em 28/04/2020; aceito em 21/09/2020; publicado na web em 16/10/2020

\begin{abstract}
USE OF A FLATBED SCANNER AND POLYURETHANE FOAM FOR QUANTIFICATION OF TOTAL IRON IN FOOD SUPLEMENTS. In this work, an alternative and low-cost technique for the extraction and quantification of $\mathrm{Fe}$ (total) from food supplements by digital image analysis is shown. The method is based on the complexation of Fe ions in solution with the 8-hydroxyquinoline ligand, forming a colored complex that is extractable from aqueous solution to polyurethane foam, coloring this one, which was initially white, to brown. After the acquisition and treatment of the digital image generated by the foam, the quantification of $\mathrm{Fe}$ is performed. Interference and recovery tests show that the method is not affected by concomitants in the sample. The method was applied to 3 different types of dietary supplements, and these results were compared to those obtained by FAAS, showing statistical equivalence. The limits of quantification obtained in this work was $1.78 \mu \mathrm{mol} \mathrm{L}^{-1}$, the limit of detection was $0.53 \mu \mathrm{mol} \mathrm{L}{ }^{-1}$, the accuracy was $97.5+4.8 \%$ and the precision was $8.45 \%$. The analytical curve was linear from 2.0 to $10.0 \mu \mathrm{mol} \mathrm{L}{ }^{-1}$, with a 2 of 0.9975 .
\end{abstract}

Keywords: digital image analysis; polyurethane foam; food supplements.

\section{INTRODUÇÃO}

As principais fontes de Ferro na dieta humana são as carnes bovina, frango, peixe, cereais, legumes, frutas e vegetais. ${ }^{1}$ Sua deficiência é tida mundialmente como a causa mais comum de anemia e é mais comum em bebês, crianças, adolescentes e gestantes, podendo ocasionar problemas no desenvolvimento cerebral e trabalho muscular. ${ }^{2,3}$ Dessa forma, é recomendado para pessoas nessa situação o uso de suplementos alimentares ricos em ferro. ${ }^{4}$ Por outro lado, o consumo excessivo desse metal pode levar a diversos problemas de saúde, como risco de câncer e doenças do fígado, doenças do coração, problemas endócrinos, artrite e diabetes. ${ }^{5,6}$

Dado o fato de que a compra de suplementos alimentares no Brasil não exige fornecimento de receita médica, e de que o público geral acredite que esse consumo seja livre de efeitos colaterais, a venda desses tem aumentando exponencialmente nos últimos anos, fazendo com que esse mercado se torne mais vulnerável a produtos de baixa qualidade. ${ }^{7}$ Nesse contexto, a determinação de ferro total em suplementos alimentares torna-se fundamental para assegurar a qualidade do produto consumido pela população.

Os métodos mais utilizados para a quantificação de ferro empregam as técnicas de espectrometria de absorção molecular no UV Vis, ${ }^{6,8-10}$ de absorção atômica (AAS), ${ }^{11-14}$ de emissão ótica em plasma indutivamente acoplado (ICP OES), ${ }^{15,16}$ técnicas eletroquímicas, ${ }^{17-19}$ e de espectrometria de massas em plasma indutivamente acoplado (ICP MS). ${ }^{20,21}$ Todas essas técnicas necessitam de alto investimento e etapas laboriosas de preparo de amostra.

Uma técnica que vem ganhando cada vez mais aplicações nas últimas décadas é a análise de imagens digitais (DIA, do inglês Digital Images Analysis). São várias as produções que podem ser citadas utilizando DIA, como análise de corantes e metais em alimentos, ${ }^{22-26}$ análises ambientais, ${ }^{27,28}$ análise de bebidas alcóolicas, ${ }^{29-31}$ análise de pesticidas, ${ }^{32,33}$ análise de ligas metálicas ${ }^{34-36}$ dentre várias outras.

Nessa técnica, empregam-se analitos com características coloridas, ou um analito que reaja com um cromogênio para produzir

*e-mail: wfpacheco@id.uff.br uma espécie colorida, seguido do registro de uma imagem, com o auxílio de uma câmera digital, câmera hiperespectral, scanner, webcam ou smartphone. A análise dessa imagem irá fornecer dados que permitem quantificar o analito. ${ }^{37-40}$

Diferentes abordagens são utilizadas nessa reação de produção de cor. É possível utilizar a própria coloração do analito em solução, ${ }^{41}$ ou situação em que analito e reagente cromogênio vão ser adicionados à solução, ${ }^{24,29}$ ou em que analito e reagente em fase líquida produzem um composto colorido que é extraído para uma fase sólida, ${ }^{25,42}$ ou ainda em que o analito é adicionado diretamente a um substrato sólido previamente modificado com o reagente (como nos dispositivos analíticos microfluídicos baseados em papel). ${ }^{33,43-46} \mathrm{Nas}$ duas primeiras situações o registro de imagem é feito diretamente em solução, enquanto nas outras em fase sólida. Esses últimos casos trazem consigo a vantagem adicional da separação do analito da matriz, possivelmente eliminando interferências.

Quanto aos dispositivos de captura de imagem, embora o uso de câmeras hiperespectrais venha aumentando nos métodos de DIA, ainda são dispositivos custosos e necessitam de tratamento mais elaborado das imagens obtidas. ${ }^{47,48}$ Já os métodos que empregam scanners ou câmeras digitais são simples e de baixo custo, oferecendo ainda a vantagem de possibilidade de miniaturização dos sistemas e realização de medidas fora de laboratório. No entanto, no caso da utilização de câmeras digitais, há como desvantagem a necessidade de um controle externo de fatores que afetem a resposta analítica, como iluminação e distância focal. Diferentes abordagens são apresentadas na literatura para resolver essa questão, como a construção de dispositivos (caixas com iluminação interna) para conter a amostra ou o uso de padrões de calibração junto às amostras. Ressalta-se que o uso de scanners de mesa torna essas preocupações desnecessárias, já que parâmetros de iluminação e focalização são fixos nestes dispositivos. ${ }^{42,49-51}$

Para realizar a quantificação da coloração são utilizados modelos matemáticos denominados espaços de cor, que usualmente baseiam-se na decomposição em cores primárias, de maneira similar à percepção humana. ${ }^{52}$ Dentre os vários espaços existentes (RGB, CIE XYZ, HSV e CIELAB $)^{52-54}$ destaca-se o espaço RGB, com grande aplicação, em 
parte devido à sua maior simplicidade..$^{55}$ Nesse sistema, a cor analisada é decomposta em cores primárias e dados são reportados em forma de coordenadas $\mathrm{R}, \mathrm{G}$ e $\mathrm{B}$, sendo $\mathrm{R}$ a intensidade para vermelho, $\mathrm{G}$ para verde e $\mathrm{B}$ para azul, variando desde a cor preto $[0,0,0]$ até branco $\left[2^{\mathrm{n}-1}, 2^{\mathrm{n}-1}, 2^{\mathrm{n}-1}\right]$, sendo $n$ o número de bits de digitalização para cada canal no dispositivo. A partir desses dados, diferentes abordagens podem ser utilizadas para cálculo do sinal analítico. A absorbância efetiva, muito empregada em diversos trabalhos presentes na literatura, ${ }^{25,26,42,49}$ é calculada como a seguir:

$$
A_{c}=-\log \frac{I_{s}}{I_{b}}
$$

Em que $A c$ é a absorbância efetiva em cada canal (vermelho, verde ou azul), $I_{s}$ é a intensidade da coloração do padrão analítico ou amostra nesse canal, e $I_{b}$ é a intensidade da referência (branco) no mesmo canal.

No presente trabalho é apresentada a determinação de ferro total em amostras de suplementos alimentares, através da complexação desse elemento com 8-hidroxiquinolina para formar um produto colorido, o qual é sorvido em espuma de poliuretano, permitindo simultaneamente a extração do complexo contendo o analito e a formação do substrato sólido colorido.

O emprego de espuma de poliuretano (EPU) como fase sólida para DIA apresenta a qualidade de ser um material de baixo custo e boa capacidade sortiva, permitindo realizar a sorção e pré concentração do analito, obtendo-se então coloração mais intensa no meio sortivo e, assim, aumentando a sensibilidade do método. ${ }^{25,51} \mathrm{~A}$ 8-hidroxiquinolina (8HQ) é um reagente cromogênio muito utilizado para funcionalização de fases sólidas com o objetivo de pré-concentrar e extrair Fe e outros metais de solução aquosa. ${ }^{56,57}$ No entanto, até aonde temos conhecimento, ela não teve seu uso explorado para DIA.

\section{PARTE EXPERIMENTAL}

\section{Instrumentação}

O scanner HP Photosmart C4780 foi empregado para a obtenção das imagens digitais, e os dados gerados foram tratados com o software gratuito ImageJ (National Institutes of Health, EUA). As imagens digitais foram adquiridas com resolução de 200 dpi.

Medidas espectrométricas de absorção atômica em chama foram realizadas para comparação entre os métodos desenvolvido e de referência com o espectrômetro Shimadzu AA 6200.

Todas as soluções empregadas nesse trabalho foram preparadas com o auxílio do sistema purificador de água Sartorius Arium Pro (Alemanha), que fornecia água deionizada com resistividade de $18,2 \mathrm{M} \Omega \mathrm{cm}$

\section{Reagentes}

Solução estoque de ferro $1,00 \mathrm{mmol} \mathrm{L}^{-1}$ foi preparada a partir de diluição de solução padrão de ferro (Merck, Alemanha) de concentração $1000 \mathrm{mg} \mathrm{L}^{-1}$. Solução estoque de 8-hidroxiquinolina de concentração $0,100 \mathrm{~mol} \mathrm{~L}^{-1}$ foi preparada a partir da solubilização do sólido (Vetec, Brasil) em acetona (Merck, Alemanha).

Tampão Britton-Robbinson (BR) $0,080 \mathrm{~mol} \mathrm{~L}^{-1}$ foi preparado como sugere a literatura ${ }^{58}$ a partir de ácido acético, bórico e fosfórico (Merck, Alemanha). Solução de cloreto de sódio foi preparada a partir de solubilização do padrão (Vetec, Brasil) em água deionizada.

A espuma de poliuretano (ORTOBOM, Brasil) empregada apresentava densidade de $0,09 \mathrm{mg} \mathrm{mm}^{-3} \mathrm{e}$ foi caracterizada como tipo poliéter por espectro de infravermelho. A espuma foi previamente misturada a etanol comercial e triturada em liquidificador. Em seguida, foi seca ao ar livre e moída com o auxílio de moinho de bolas, a fim de aumentar sua área superficial e homogeneidade e, assim, melhorar a reprodutibilidade dos resultados.

\section{Procedimento experimental}

\section{Obtenção do sinal analítico}

Os experimentos relativos ao método proposto foram realizados transferindo-se para balões volumétricos de $10,00 \mathrm{~mL}$ alíquotas da solução padrão contendo ferro, o ligante 8-Hidroxiquinolina em concentração final de $1,00 \mathrm{mmol} \mathrm{L}^{-1}$ e o tampão Britton Robinson em concentração final de $8,00 \mathrm{mmol} \mathrm{L}^{-1} \mathrm{em} \mathrm{pH} \mathrm{4,0.} \mathrm{Em} \mathrm{seguida,} \mathrm{essa}$ solução era vertida em tubo de polipropileno contendo $50 \mathrm{mg}$ da EPU. A mistura era então agitada em mesa por 45 minutos a $100 \mathrm{rpm}$. Por fim era filtrada a vácuo, utilizando-se uma ponteira plástica cortada como molde. As imagens digitais das EPU contendo o complexo eram adquiridas com o auxílio de um scanner e tratadas pelo software Image $J$. Na Figura 1 encontra-se um fluxograma elucidativo desse procedimento.

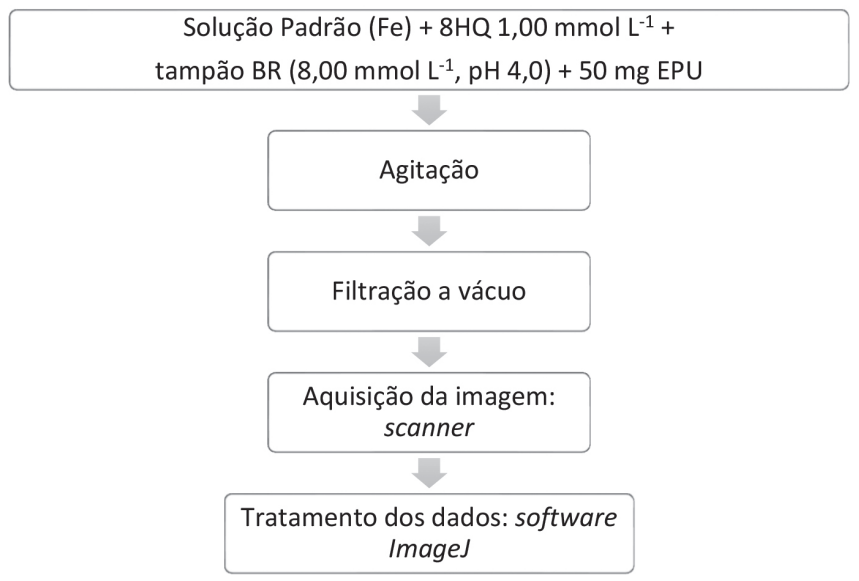

Figura 1. Esquema com as etapas do procedimento experimental proposto

A média dos valores obtidos no canal azul, que é a coloração complementar ao amarronzado observado nas EPU, é tomada como sinal analítico, permitindo obter-se um sinal de absorbância efetiva ao se calcular o logaritmo da razão entre o valor obtido para a espuma em que foi retida apenas uma fração do ligante, sem conter o analito, equivalente ao branco analítico; e o valor para outra espuma em que foi retido o complexo.

\section{Preparo das amostras para o método proposto}

Neste trabalho foram utilizadas 3 amostras diferentes, codificadas aqui como amostras I, amostra II e amostra III. Elas possuíam, segundo a bula, 10, 20 e $80 \mathrm{mg}$ de ferro (total) por comprimido, respectivamente, e cada comprimido pesava $1 \mathrm{~g}$. Os comprimidos de suplementos minerais foram macerados com graal e pistilo, de modo que $51 \mathrm{mg}$ de cada uma destas amostras foram misturados a 20,0 mL de solução de $\mathrm{HNO}_{3}\left(\right.$ Qhemis, Brasil)/ $\mathrm{H}_{2} \mathrm{O}$ 1:1. Essas misturas foram aquecidas à secura, e os sólidos residuais solubilizados em água deionizada, transferidos para balões volumétricos de 100,0 mL e avolumados até a marca também com água deionizada. Essas soluções foram chamadas de amostras mãe, ainda não estavam na forma adequada de obtenção do sinal analítico.

Para converter o ferro no complexo e permitir a medição do sinal analítico era necessário transferir alíquota da solução amostra mãe para outro recipiente, permitindo a reação com o ligante no $\mathrm{pH}$ adequado. Para conseguir isso, diferentes alíquotas de cada solução 
amostra mãe $(100,200$ e $400 \mu \mathrm{L}$, respectivamente das soluções referentes as amostras I, II e III) foram retiradas e transferidas, cada uma, para um balão volumétrico de $100 \mathrm{~mL}$. Cada um desses balões teve seu volume completado de forma a ter $1,00 \mathrm{mmol} \mathrm{L}^{-1} \mathrm{de}$ 8-hidroxiquinolina e 8,00 $\mathrm{mmol} \mathrm{L}^{-1}$ do tampão Britton Robinson em pH 4,0. Após essas soluções terem sido vertidas sobre EPU, agitadas e compactadas, as imagens geradas foram analisadas segundo a técnica de análise de imagens digitais.

\section{Construção das curvas analíticas e de adição de padrão}

Os padrões de ferro empregados na construção das curvas analíticas foram preparados a partir de diluição apropriada da solução estoque de ferro $1,00 \mathrm{mmol} \mathrm{L^{-1 }}$ em balões volumétricos de $10,00 \mathrm{~mL}$, contendo também 1,00 $\mathrm{mmol} \mathrm{L}^{-1}$ de 8-hidroxiquinolina e $8,00 \mathrm{mmol} \mathrm{L}^{-1}$ do tampão Britton Robinson em pH 4,0. Padrões analíticos de concentrações equivalentes a 0,$0 ; 2,0 ; 4,0 ; 6,0 ; 8,0$ e $10,0 \mu \mathrm{mol} \mathrm{L}{ }^{-1} \mathrm{de}$ ferro foram assim preparados. Posteriormente essas soluções eram misturadas a $50 \mathrm{mg}$ da EPU em tubo de polipropileno sob agitação em mesa por 45 minutos a $100 \mathrm{rpm}$. Como descrito anteriormente, eram então filtradas a vácuo e as imagens digitais adquiridas.

Para a construção das curvas de adição de padrão, foram adicionados para cada amostra, uma série de soluções de $10 \mathrm{~mL}$ contendo volume constante da solução amostra mãe, volume constante de ligante e tampão (de modo a ter $1,00 \mathrm{mmol} \mathrm{L}^{-1}$ de 8-hidroxiquinolina e $8,00 \mathrm{mmol} \mathrm{L}^{-1}$ do tampão Britton Robinson em $\mathrm{pH} 4,0$ ) e volume crescente de padrão de ferro (concentração adicionada de 0 a $6,00 \times 10^{-3} \mathrm{~mol} \mathrm{~L}^{-1}$ ). Vale ressaltar que o volume de solução amostra mãe foi diferente para cada tipo de amostra (100, 200 e $400 \mu \mathrm{L}$ referentes às amostras I, II e III), uma vez que tinham concentrações esperadas muito diferente.

\section{Preparo das amostras para o método de referência}

O método tomado como referência foi o emprego da técnica de espectrometria de absorção atômica em chama (F AAS). Os comprimidos de suplementos minerais foram igualmente macerados com graal e pistilo, e também $51 \mathrm{mg}$ de cada uma das mesmas amostras foram misturados a 20,0 mL de solução de $\mathrm{HNO}_{3}$ (Qhemis, Brasil)/ $\mathrm{H}_{2} \mathrm{O}$ 1:1. As misturas foram aquecidas à secura, e os sólidos residuais solubilizados em água deionizada, transferidos para balões volumétricos de $100,0 \mathrm{~mL}$ e as soluções avolumadas até a marca também com água deionizada.

Alíquotas de $1000 \mu \mathrm{L}$ foram retiradas para a análise da amostra I e de $5000 \mu \mathrm{L}$ para análise das amostras II e III. Essas alíquotas foram inseridas em balões volumétricos de $10,00 \mathrm{~mL}$ e avolumadas com água deionizada, sendo as soluções diluídas resultantes quantificadas diretamente por F AAS.

Validação do método, cálculo dos parâmetros analíticos de mérito e estudos de possíveis interferências

Para garantir que o método analítico aqui proposto gere informações confiáveis é necessário seguir um protocolo de validação. No presente trabalho, foi seguido o guia de validação de métodos analíticos da IUPAC. ${ }^{59}$ Os parâmetros avaliados neste estudo foram: exatidão, precisão, linearidade, limite de detecção, limite de quantificação e avaliação da matriz.

A precisão das medições foi dada pela dispersão dos resultados obtidos em condições semelhantes e calculada como o desvio padrão relativo dos sinais analíticos provenientes de dez diferentes espumas contendo a mesma concentração de analito (Fe 6,00 $\left.\mu \mathrm{mol} \mathrm{L}^{-1}\right)$.

A exatidão foi determinada por meio de testes de recuperação empregando-se amostras de suplementos alimentares. Alíquotas de $200 \mu \mathrm{L}$ das soluções diluídas das amostras foram submetidas em triplicatas ao método proposto. Em seguida, triplicatas de alíquotas de mesmo volume dessas amostras foram fortificadas $\operatorname{com} 2,00 \mu \mathrm{mol} \mathrm{L} \mathrm{L}^{-1}$ de Ferro (III) e a recuperação obtida utilizada como a exatidão.

Os limites de detecção $\left(\mathrm{S}_{\mathrm{LD}}\right)$ e quantificação $\left(\mathrm{S}_{\mathrm{LQ}}\right)$ foram determinados, respectivamente, pelos critérios $\mathrm{S}_{\mathrm{LD}}=\mathrm{Yb}+3 \mathrm{Sb}$ e $\mathrm{S}_{\mathrm{LQ}}=\mathrm{Yb}+10 \mathrm{Sb}$, sendo $\mathrm{Yb}$ o sinal analítico do branco (ou o intercepto da curva analítica) e Sb o desvio padrão para o sinal analítico obtido para dez soluções $6,00 \mu \mathrm{mol} \mathrm{L} \mathrm{L}^{-1}$ de Ferro (III). ${ }^{60}$

A fim de avaliar a possibilidade de concomitantes presentes nas amostras de suplementos causarem interferência no sinal analítico, espécies consideradas como possíveis interferentes foram selecionadas tendo em vista a composição esperada segundo as informações fornecidas nos rótulos das amostras.

Para avaliar o efeito de possíveis interferências aditivas, o sinal de absorbância obtido para uma solução contendo ferro em concentração equivalente à média prevista pelos rótulos dos suplementos alimentares analisados foi comparado ao sinal do analito gerado na presença dos íons Ca II, Cu II, Mg II e Zn II, individualmente e em conjunto. A Tabela 1 mostra as concentrações das espécies presentes nas soluções analisadas nesse estudo.

Tabela 1. Concentrações das espécies avaliadas como possíveis interferentes espectrais, segundo previsto pelos rótulos das amostras

\begin{tabular}{ccc}
\hline Solução & {$\left[\mathrm{Fe} \mathrm{III]}\left(\mu \mathrm{g} \mathrm{L}^{-1}\right)\right.$} & [interferente] $\left(\mu \mathrm{g} \mathrm{L}^{-1}\right)$ \\
\hline Fe III & 336 & ------ \\
Fe III + Ca II & 336 & 1700 \\
Fe III + Cu II & 336 & 3,1 \\
Fe III + Mg II & 336 & 816 \\
Fe III + Zn II & 336 & 47,6 \\
\hline
\end{tabular}

Para avaliar a ocorrência de interferências não aditivas, comparações entre coeficientes angulares de curvas analíticas e de adição de analito geradas com as amostras estudadas neste trabalho (construídas conforme relatado no item 2.3) foram realizadas. Tais dados foram gerados em triplicatas.

\section{RESULTADOS E DISCUSSÃO}

\section{Informações preliminares}

A abordagem escolhida para quantificar Fe total pela técnica de análise de imagens digitais necessita da formação de um complexo colorido, e que este complexo possua a capacidade de ser sorvido sobre uma fase sólida, para posteriormente ter sua imagem registrada. O reagente cromogênio 8-hidroxiquinolina (8HQ) foi escolhido pelo fato de apresentar capacidade de complexar com os íons Fe III (Log $\beta=36,9)$ muito superior a outros metais. ${ }^{61-67} \mathrm{~A}$ fase sólida selecionada foi espuma de poliuretano (EPU), dada sua grande capacidade sortiva para espécies hidrofóbicas. ${ }^{68,69} \mathrm{O}$ complexo formado entre Fe III e 8HQ apresentou coloração marrom quando sorvido sobre a EPU, e estudos preliminares mostraram uma que a intensidade de coloração aumentava proporcionalmente com o aumento da concentração, sem variações ao longo do tempo (durante 3 horas).

A literatura ${ }^{61}$ aponta que a estequiometria dessa reação é de 1 para 3, de forma que ocorre em solução:

$$
\mathrm{Fe}^{3+}{ }_{(\mathrm{aq})}+3\left(8-\mathrm{HQ}^{-}\right)_{(\mathrm{aq})} \leftrightarrows\left[\mathrm{Fe}(8-\mathrm{HQ})_{3}\right]_{(\mathrm{aq})}
$$

Assim, de modo a alcançar-se máxima sensibilidade, no presente 
trabalho foram estudadas e otimizadas as condições de formação do complexo Fe(III)-8HQ e sua sorção em EPU, em termos dos parâmetros concentração do ligante, $\mathrm{pH}$ e força iônica do meio, tempo de agitação e massa de fase sólida. Para todos os experimentos a velocidade de agitação foi fixada em 100 rpm, velocidade máxima oferecida pelo aparato utilizado.

Vale ressaltar que o preparo das amostras, envolvendo a dissolução em solução concentrada de $\mathrm{HNO}_{3}$, ocasiona a conversão de íons Fe (II) possivelmente presentes em Fe (III) como descrito na seguinte equação. ${ }^{70,71}$ É justamente essa etapa que garante que o método seja para ferro total e não Fe(III).

$3 \mathrm{Fe}^{2+}+\mathrm{NO}_{3}^{-}+4 \mathrm{H}^{+} \rightleftharpoons 3 \mathrm{Fe}^{3+}+\mathrm{NO}+2 \mathrm{H}_{2} \mathrm{O} \quad \Delta \mathrm{E}=0,186 \mathrm{~V}$

\section{Efeito do tempo de agitação e massa de EPU}

As variáveis tempo de agitação e massa de EPU empregada foram avaliadas simultaneamente, a fim de averiguar se o aumento da massa afetaria a magnitude do sinal analítico. Nesse estudo, a massa de EPU foi estudada em 50, 75 e $100 \mathrm{mg}$, enquanto o tempo de agitação entre 15 e 150 minutos, com concentração de ferro fixada em $6,00 \mu \mathrm{mol} \mathrm{L}^{-1}$, 10,0 mmol L-1 8 HQ e 8,00 mmol L-1 BR pH 6,0.

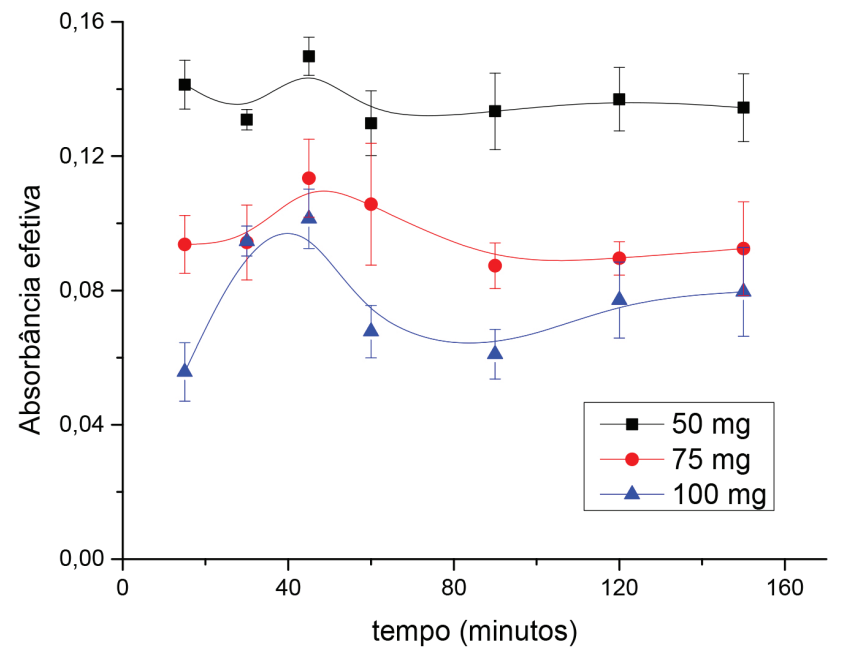

Figura 2. Efeito do tempo de agitação e massa de EPU sobre o sinal analítico (6,00 $\mu \mathrm{mol} \mathrm{L}{ }^{-1} \mathrm{Fe} ; 1,00 \mathrm{mmol} \mathrm{L}^{-1} 8 \mathrm{HQ} ; 8,00 \mathrm{mmol} \mathrm{L}^{-1} \mathrm{BR} \mathrm{pH} \mathrm{6,0)}$

Avaliando-se os resultados obtidos neste experimento, mostrados na Figura 2, foi observado que um aumento da massa ocasionou diminuição na intensidade do sinal analítico, uma vez que quanto maior a dispersão do complexo no meio sorvente, menor o sinal alcançado. Para a menor massa estudada $(50 \mathrm{mg})$, o sinal de absorbância é mais elevado, já que a mesma quantidade de complexo é retida numa área superficial menor, fato também observado em outros trabalhos da literatura que envolvem retenção de complexos em fases sólidos com quantificação por DIA. ${ }^{25,26,51}$

Quanto ao tempo de agitação, não foi observada significativa influência deste sobre o sinal analítico. Assim, com o intuito de garantir máxima sensibilidade às determinações, foi selecionada a massa de $50 \mathrm{mg}$ de EPU e tempo de agitação de 45 minutos.

\section{Efeito da concentração de $8 \mathrm{HQ}$}

O estudo da concentração de 8HQ sobre o sistema foi avaliado no intervalo de $1,00 \times 10^{-3} \mathrm{mmol} \mathrm{L}^{-1}$ a $10 \mathrm{mmol} \mathrm{L}^{-1}$, com concentração de ferro fixada em $6,00 \mu \mathrm{mol} \mathrm{L} \mathrm{L}^{-1}$. O resultado desse estudo é apresentado na Figura 3.

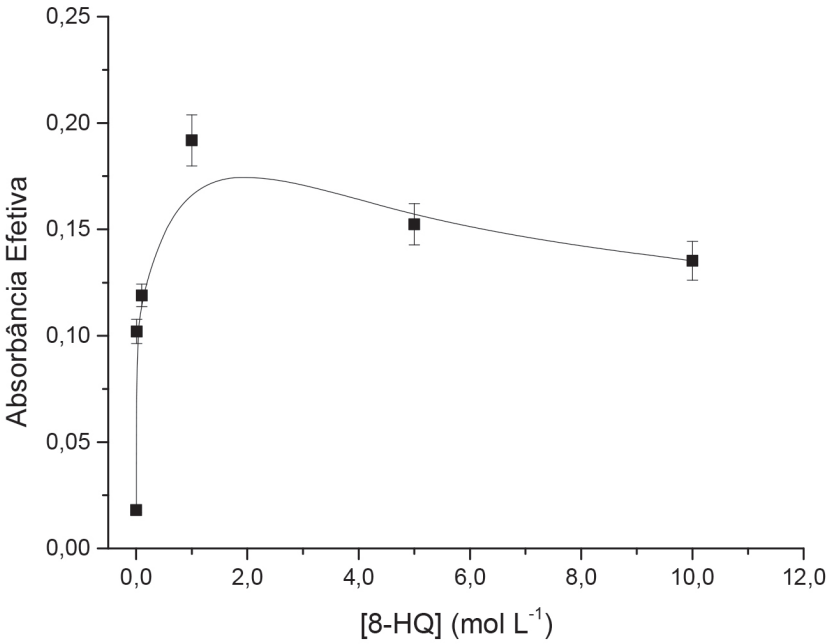

Figura 3. Efeito da concentração do ligante sobre o sinal analítico (6,00 $\mu \mathrm{mol} \mathrm{L} \mathrm{L}^{-1} \mathrm{Fe} ; 8,00 \mathrm{mmol} \mathrm{L}^{-1} \mathrm{BR} \mathrm{pH} \mathrm{6,0;} 50 \mathrm{mg}$ EPU; 45 minutos de agitação)

Maiores concentrações do ligante aumentam inicialmente a concentração de complexo gerado e retido na EPU, tornando mais intenso o sinal de absorbância. Observa-se, contudo, um decréscimo do sinal nas concentrações mais elevadas de $8 \mathrm{HQ}$. Isso ocorre possivelmente por conta da retenção de fração do ligante livre sobre a EPU em elevadas concentrações deste, provocando diminuição do sinal analítico. Assim sendo, selecionou-se a concentração de 1,00 mmol L-1 de $8 \mathrm{HQ}$ para ser empregada nos experimentos subsequentes. Tal concentração oferece sinal satisfatório dispensando o uso de quantidades excessivas do ligante.

\section{Efeito do pH do meio reacional}

$\mathrm{O}$ pH do meio foi também considerado um fator importante a ser avaliado tendo em vista que tanto ferro quanto o ligante podem se apresentar como diferentes espécies graças às suas características ácido/básicas. $\mathrm{O}$ pH das soluções foi ajustado utilizando-se o tampão Britton Robinson, composto pelos sistemas acetato, fosfato e borato, cada um em concentração de $8,00 \mathrm{mmol} \mathrm{L}^{-1}$, e variado entre $\mathrm{pH} 2,34$ e 12,4, fixadas as concentrações de ferro em $6,00 \mu \mathrm{mol} \mathrm{L}^{-1}$ e de $8 \mathrm{HQ}$ em 1,00 mmol L'-1. Adicionalmente, uma solução contendo ferro e $8 \mathrm{HQ}$ nessas concentrações, sem ajuste de $\mathrm{pH}$, foi avaliada. O resultado desse estudo é apresentado na Figura 4.

Pode-se observar um aumento no sinal analítico entre $\mathrm{pH} 2$ e 5 , uma queda até $\mathrm{pH} 8$, e um platô a partir de então. Esse comportamento pode ser explicado em função do equilíbrio ácido base do sistema, envolvendo a carga superficial do sorvente (EPU), a formação do complexo e de hidróxidos de ferro.

Em meio excessivamente ácido ( $\mathrm{pH}$ inferior a 2,0), a superfície do sólido encontra-se protonada, ${ }^{69}$ e essa carga positiva torna desfavorável à sorção do complexo. A medida que o pH aumenta, diminui a carga superficial do sólido até sua neutralização, ao mesmo tempo que aumenta a tendência de formação do complexo, visto que 8 HQ apresenta pKa $6,50 \pm 0,20 .{ }^{72}$

Em $\mathrm{pH}$ superior a 6,0 o processo de sorção do complexo sobre a EPU é prejudicado pelo fato da espécie Fe III não ser predominante no meio reacional. Nesse intervalo mais alcalino, há formação de hidróxidos de ferro, que tornam este metal indisponível para a complexação com 8HQ, resultando em queda do sinal analítico. ${ }^{62,73-75}$

Sinais analíticos mais intensos foram alcançados em pH 4,01. Pelos resultados expostos na Figura 4 é possível notar que em pH 5,7, obtido seja com tamponamento ou não (solução sem ajuste de $\mathrm{pH}$ ), 


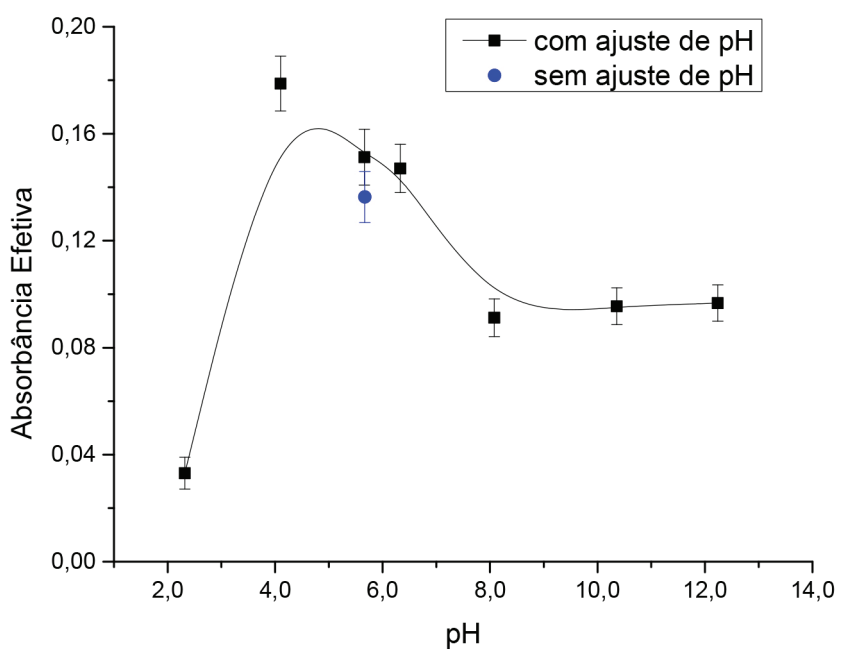

Figura 4. Efeito do pH do meio sobre o sinal analítico (Fe 6,00 $\mu \mathrm{mol} \mathrm{L}^{-1}$; $8 H Q$ 1,00 mmol $L^{-1} ; 8,00 \mathrm{mmol} \mathrm{L}^{-1} \mathrm{BR} ; 50 \mathrm{mg}$ EPU; 45 minutos de agitação)

a intensidade do sinal alcançado é a mesma, o que leva a crer que apenas o pH interfere no sinal analítico, e não a força iônica do meio reacional, no intervalo avaliado.

\section{Efeito da força iônica do meio reacional}

A fim de confirmar a hipótese levantada anteriormente e avaliar se a ausência de influência da força iônica era ou não limitada ao intervalo de concentração do tampão estudado $\left(8,00 \mathrm{mmol} \mathrm{L}^{-1}\right)$, foi realizado um estudo mantendo-se constante o $\mathrm{pH}$ e variando-se a força iônica do meio.

Espumas contendo a mesma concentração de complexo $\mathrm{Fe}$ (III)-8HQ foram avaliadas sob diferentes ajustes de força iônica por adição de $\mathrm{KNO}_{3}$, cuja concentração foi variada entre 0 e $2,0 \mathrm{~mol} \mathrm{~L}^{-1}$, fixadas as concentrações de ferro em $6,00 \mu \mathrm{mol} \mathrm{L^{-1 }}$, de $8 \mathrm{HQ}$ em $1,00 \mathrm{mmol} \mathrm{L}^{-1}$ e de tampão BR em 8,00 mmol L-1 e pH 4,0.

Após avaliar as imagens digitais geradas, a baixa variabilidade dos dados de absorbância obtidos comprovou que a força iônica é um parâmetro pouco significativo à intensidade do sinal analítico.

\section{Avaliação de possíveis interferências}

Como mencionado anteriormente, as espécies consideradas como possíveis interferentes ao método foram selecionadas tendo em vista a composição esperada segundo as informações fornecidas pelos rótulos das amostras de suplementos alimentares (sais de cálcio, cobre II, magnésio e zinco). As concentrações das espécies presentes nas soluções analisadas neste estudo foram mencionadas no item 2.4.

A Figura 5 mostra a variação do sinal de absorbância do complexo Fe(III)-8HQ mediante a adição destes íons.

Nota-se que os desvios para as soluções contendo o analito e cada metal individualmente, bem como para a solução contendo todos os metais e o analito são inferiores a 5\% do sinal do ferro isoladamente, indicando que as espécies estudadas não interferem no sinal analítico nas concentrações esperadas nas amostras. Embora todos os metais avaliados tenham a capacidade de formar complexos com a 8HQ, a constante de formação global do complexo Fe(III)-8HQ é significativamente maior que dos demais complexos com este ligante..$^{62,72}$

Para avaliar a influência de interferências não aditivas ao método proposto, previamente à sua aplicação nas amostras estudadas, foram comparados os coeficientes angulares obtidos pela técnica de calibração externa com a obtida pela técnica de adição padrão,

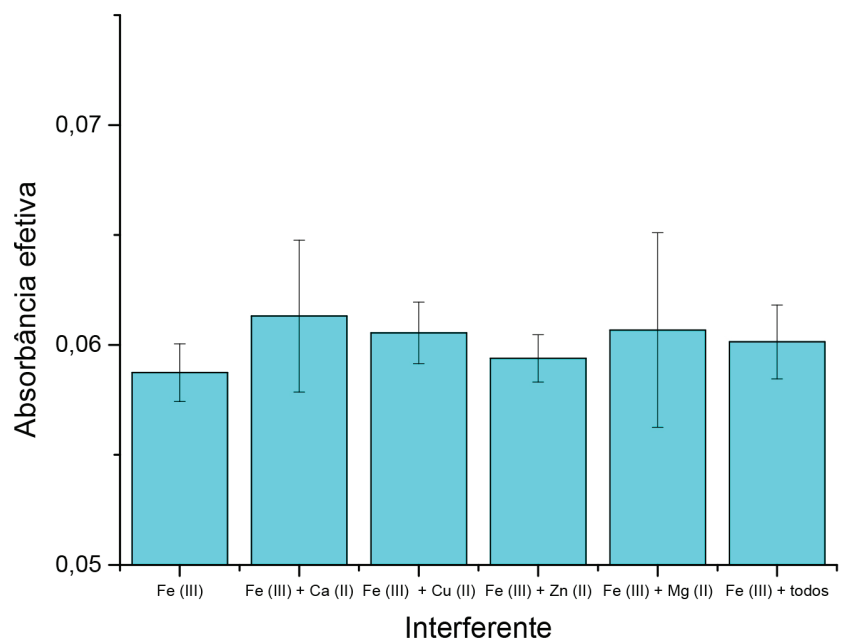

Figura 5. Influência de alguns íons sobre o sinal do Fe III (1,00 mmol L $\mathrm{L}^{-1} 8 \mathrm{HQ}$; 8,00 $\mathrm{mmol} \mathrm{L}^{-1} \mathrm{BR} \mathrm{pH}$ 4,0; $50 \mathrm{mg}$ EPU; 45 minutos de agitação)

(construídas conforme relatado no item 2.4). O resultado desse estudo é apresentado na Tabela 2.

Tabela 2. Comparação entre coeficientes angulares das curvas analíticas e de adição de analito $\left(\mathrm{F}_{\text {crítico }}=9,28 ; \mathrm{T}_{\text {crítico }}=2,78\right)^{60}$

\begin{tabular}{ccccc}
\hline \multirow{2}{*}{ Amostra } & \multicolumn{2}{c}{ Coeficiente angular } & & \\
\cline { 2 - 3 } & Calibração externa & Adição Padrão & & \\
\hline I & $0,0185 \pm 0,0012$ & $0,0208 \pm 0,0006$ & 0,28 & 2,03 \\
II & $0,0180 \pm 0,0007$ & $0,0188 \pm 0,0004$ & 2,28 & 1,15 \\
III & $0,0191 \pm 0,0005$ & $0,0201 \pm 0,0010$ & 0,25 & 1,13 \\
\hline
\end{tabular}

A comparação dos coeficientes angulares foi feita por meio do teste t-Student, utilizando-se as médias das inclinações obtidas para três curvas analíticas e três curvas de adição de analito referentes a cada amostra. Antes da aplicação do teste de comparação entre médias, um teste $\mathrm{F}$ para comparação entre as variâncias foi realizado a fim de verificar se havia diferença estatística entre elas.

Para todas as amostras avaliadas, $\mathrm{F}_{\text {calculado }}<\mathrm{F}_{\text {crítico }}$ no nível de confiança de $95 \%$, o que permite concluir que não há diferença estatística entre a precisão das médias em cada caso. Portanto, é possível aplicar o teste t-Student de comparação entre médias utilizando-se a variância agrupada. A hipótese nula é mantida para estas amostras no nível de confiança de $95 \%$, pois $\mathrm{T}_{\text {calculado }}<\mathrm{T}_{\text {crítico. }}{ }^{60}$ Assim, é possível afirmar que os concomitantes presentes na matriz estudada não causam interferência ao método.

\section{Validação e parâmetros analíticos de mérito do método}

Os parâmetros analíticos de mérito foram obtidos nas condições otimizadas, descritas anteriormente. Nessas condições, uma curva analítica típica apresenta a equação Abs $=0,0112\left[\mathrm{Fe}, \mu \mathrm{mol} \mathrm{L} \mathrm{L}^{-1}\right]+0,0132 \mathrm{com} \mathrm{r}^{2}=0,9975$. Para comprovar a linearidade, foi feita uma ANOVA. O teste mostrou que a linearidade foi significativa enquanto a falta de ajuste foi não significativa. A curva analítica está representada na Figura 6A, e as imagens utilizadas para construí-la na Figura 6B. A região de interesse (ROI) usada para captar o sinal constituía de uma fração da imagem, diferentes circunferências, desde que menor que o raio da figura, não influenciavam no sinal. Como descrito no item 2.4 , foram calculados os limites de detecção $\left(0,53 \mu \mathrm{mol} \mathrm{L}^{-1}\right)$ e de quantificação $\left(1,78 \mu \mathrm{mol} \mathrm{L} \mathrm{L}^{-1}\right)$. A precisão do método foi de $8,45 \%$, enquanto que 
Tabela 3. Métodos para quantificação de Ferro por DIA

\begin{tabular}{|c|c|c|c|c|}
\hline Referência & Dispositivo & Amostra & Reagente & LOD/LOQ \\
\hline 76 & Câmera digital & Aquosa sintética & $\mathrm{KSCN}$ & $300 \mu \mathrm{g} \mathrm{L}^{-1}$ (LOQ) \\
\hline 28 & $\begin{array}{l}\text { Câmera digital, scanner, } \\
\text { smartphone }\end{array}$ & $\begin{array}{c}\text { Águas doces, solos, sementes, } \\
\text { folhas, carnes }\end{array}$ & $\begin{array}{c}\text { 1,10-fenantrolina; } \\
\text { 2,4,6-tris(2-piridil)-s-triazina; } \\
\text { salicilato de sódio }\end{array}$ & $\begin{array}{c}30-3440 \mathrm{mg} \mathrm{L}^{-1}(\mathrm{LOD}), \\
100-11500 \mathrm{mg} \mathrm{L}^{-1} \text { (LOQ) }\end{array}$ \\
\hline 23 & scanner & Águas doces e folhas & 1,10-fenantrolina & $\begin{array}{l}14,1 \mu \mathrm{g} \mathrm{L}^{-1} \text { (LOD), } \\
46,5 \mu \mathrm{g} \mathrm{L}^{-1} \text { (LOQ) }\end{array}$ \\
\hline 77 & Smartphone & Águas doces e solos & 1,10-fenantrolina & $90 \mu \mathrm{g} \mathrm{L}^{-1}(\mathrm{LOD})$ \\
\hline 78 & Webcam & Vinho branco & 1,10-fenantrolina & $\begin{array}{l}42 \mathrm{~g} \mathrm{~L}^{-1} \text { (LOD), } \\
141 \mathrm{~g} \mathrm{~L}^{-1} \text { (LOQ) }\end{array}$ \\
\hline Presente trabalho & scanner & Suplemento alimentar & 8-hidroxiquinolina & $\begin{array}{l}59 \mu \mathrm{g} \mathrm{L}^{-1} \text { (LOD), } \\
195 \mu \mathrm{g} \mathrm{L}^{-1}(\mathrm{LOQ})\end{array}$ \\
\hline
\end{tabular}

(a)

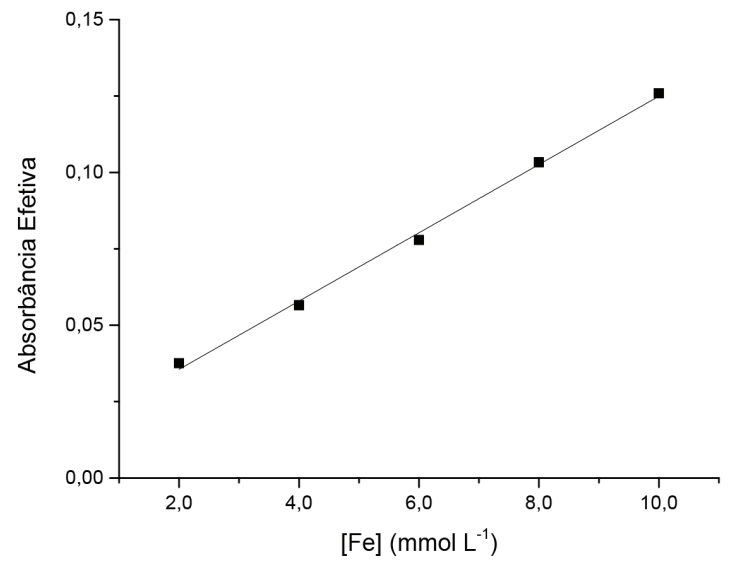

(b)
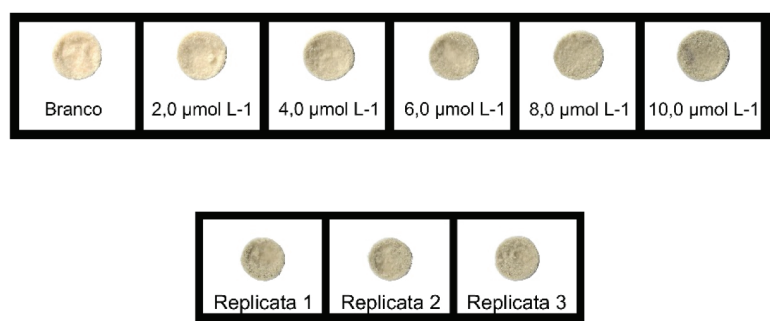

Figura 6. (a) Curva analítica típica (b) Exemplo de imagem digital gerada para uma curva analítica e amostra analisada

a exatidão apresentou o valor de $97,5 \pm 4,8 \%$, parâmetros esses calculados segundo o que foi proposto no item 2.4.

Ao comparar os limites do método proposto com outros já presentes na literatura para a determinação de ferro por DIA (Tabela 3), concluiu-se que os limites aqui apresentados são semelhantes ou melhores que os demais. Ressalta-se ainda que nenhum dos métodos já propostos empregaram a complexação com o ligante 8HQ ou a extração do analito para um suporte sólida, de modo a promover seu isolamento da matriz da amostra e pré-concentração.

\section{Aplicação do método e comparação com método de referência}

A exatidão do método proposto foi avaliada tanto pela realização de testes de recuperação quanto pela comparação estatística dos resultados obtidos para as quantificações com um método de referência de FAAS (Tabela 4). Todas as amostras foram avaliadas em triplicatas. De fato, os teores de ferro total determinados se encontraram em acordo com o que era proposto no rótulo dos suplementos.
Tabela 4. Quantificações das amostras de suplementos alimentares empregando DIA e FAAS $\left(\mathrm{F}_{\text {crítico }}=9,28 ; \mathrm{T}_{\text {crítico }}=2,78\right)^{60}$

\begin{tabular}{ccccc}
\hline Amostra & DIA $\left(\mathrm{mg} \mathrm{g}^{-1}\right)$ & F AAS $\left(\mathrm{mg} \mathrm{g}^{-1}\right)$ & $\mathrm{F}_{\text {calculado }}$ & $\mathrm{T}_{\text {calculado }}$ \\
\hline I & $78,0 \pm 5,8$ & $90,0 \pm 6,6$ & 1,32 & 1,59 \\
II & $25,9 \pm 3,4$ & $22,9 \pm 1,3$ & 6,81 & 0,97 \\
III & $10,0 \pm 1,0$ & $9,5 \pm 0,8$ & 1,70 & 0,44 \\
\hline
\end{tabular}

Após a aplicação de teste t-Student com equivalência estatística da precisão no nível de confiança de $95 \%$ para efetuar a comparação entre estes últimos resultados, foi possível concluir que a diferença entre as concentrações determinadas ocorre devido a erros aleatórios. ${ }^{60}$ Em outras palavras, não há diferença estatística entre os resultados apresentados. Essa equivalência estatística juntamente com os elevados percentuais de recuperação alcançados para ambas as amostras permitem concluir que o método apresenta exatidão satisfatória. ${ }^{79}$

\section{CONCLUSÕES}

Os resultados expostos confirmam que o método aqui proposto é simples, exato e preciso. Espécies frequentemente presentes nas amostras de suplementos alimentares analisadas, como íons cálcio, cobre II, magnésio e zinco não interferem no método aqui proposto. De fato, a retenção do analito sob a forma de complexo com o ligante 8HQ sobre EPU promove um processo de extração, de modo a realizar seu isolamento da matriz da amostra, evitando a ocorrência de interferências.

Os resultados das quantificações de ferro total por ambas as metodologias, proposta e de referência (F AAS), mostraram-se estatisticamente equivalentes. Todavia, o método elaborado ao longo deste trabalho emprega aparatos de baixo custo (um scanner como detector e um software de domínio público para obtenção do sinal analítico), o que viabiliza a sua implementação.

\section{AGRADECIMENTOS}

Os autores são gratos às seguintes instituições pelo fornecimento de bolsas e projetos de pesquisa: Fundação de Amparo à Pesquisa do Estado do Rio de Janeiro (FAPERJ), Conselho Nacional de Desenvolvimento Científico e Tecnológico (CNPq) e Coordenação de Aperfeiçoamento de Pessoal de Nível Superior (CAPES).

\section{REFERÊNCIAS}

1. Lieu, P. T.; Heiskala, M.; Peterson, P. A.; Yang, Y.; Mol. Aspects Med. 2001, 22, 1 . 
2. Abe-Matsumoto, L. T.; Sampaio, G. R.; Bastos, D. H. M.; Cad. Saude Publica 2015, 31, 1371 .

3. Biesalski, H. K.; Tinz, J.; Nutrition 2017, 36, 60.

4. World Health Organization (WHO); The role of iron in human metabolic processes, 2011.

5. Niederau, C.; Fischer, R.; Purschel, A.; Stremmel, W.; Haussinger, D.; Gastroenterology 1996, 110, 1107.

6. Kassem, M. A.; Amin, A. S.; Food Chem. 2013, 141, 1941.

7. Fibigr, J.; Šatínský, D.; Solich, P.; Anal. Chim. Acta 2018, 1036, 1.

8. Almendral, M. J.; Alonso, A.; Porras, M. J.; Garcı, M. A.; Curto, Y.; Microchim. Acta 2004, 147, 117.

9. Hasani, M.; Rezaei, A.; Abdollahi, H.; Spectrochim. Acta, Part A 2007, $68,414$.

10. Ruengsitagoon, W.; Talanta 2008, 74, 1236.

11. Cristina, M.; Quaresma, B.; Cassella, R. J.; De, M.; Santelli, R. E.; Talanta 2004, 62, 807.

12. Suzuki, T.; Sensui, M.; Anal. Chim. Acta 1991, 245, 43.

13. Niemelä, M.; Kola, H.; Eilola, K.; Perämäki, P.; J. Pharm. Biomed. Anal. 2004, 35, 433

14. da Silva, J. E.; da Silva, F. A.; Pimentel, M. F.; Saldanha, R.; Lins, V.; Montenegro, B. S. M.; Ara, A. N.; Conceic, M.; Talanta 2006, 70, 522.

15. Braunschweig, J.; Bosch, J.; Heister, K.; Kuebeck, C.; Meckenstock, R. U.; J. Microbiol. Methods 2012, 89, 41.

16. Algeo, J. D.; Heine, D. R.; Phillips, H. A.; Hoek, F. B. G.; Schneider, M. R.; Freelin, J. M.; Denton, M. B.; Spectrochim. Acta 1985, 4, 1447.

17. Laglera, L. M.; Monticelli, D.; Environ. Electrochem. 2017, 3, 123.

18. Lin, M.; Pan, D.; Hu, X.; Han, H.; Li, F.; Sensors Actuators, B 2015 , $219,164$.

19. Li, F.; Pan, D.; Lin, M.; Han, H.; Hu, X.; Kang, Q.; Electrochim. Acta 2015, 176, 548

20. Wang, S.; Ouerdane, L.; Hoekenga, O.; Szpunar, J.; Lobinski, R.; Food Chem. 2019, 294,

414.

21. Merli, D.; Ravasio, F.; Protti, S.; Pesavento, M.; Profumo, A.; Talanta 2014, 130,90

22. Sorouraddin, M. H.; Saadati, M.; Mirabi, F.; J. Food Drug Anal. 2015, $23,447$.

23. Peng, B.; Chen, G.; Li, K.; Zhou, M.; Zhang, J.; Zhao, S.; Food Chem. 2017, 230, 667 .

24. Vidal, M.; Garcia-Arrona, R.; Bordagaray, A.; Ostra, M.; Albizu, G.; Talanta 2018, 184, 58.

25. de Sá, I. C.; Feiteira, F. N.; Pacheco, W. F.; Food Anal. Methods 2020, 13, 962.

26. De O. Costa, G.; Feiteira, F. N.; De M. Schuenck, H.; Pacheco, W. F.; Anal. Methods 2018, 10, 4463.

27. Saraji, M.; Bagheri, N.; Sensors Actuators, B 2018, 270, 28.

28. Mohamed, A. A.; Shalaby, A. A.; Food Chem. 2019, 274, 360.

29. Curbani, L.; Gelinski, J. M. L. N.; Borges, E. M.; Food Anal. Methods 2020, 13, 249 .

30. Pessoa, K. D.; Suarez, W. T.; dos Reis, M. F.; de Oliveira Krambeck Franco, M.; Moreira, R. P. L.; dos Santos, V. B.; Spectrochim. Acta, Part A 2017, 185, 310 .

31. Tôrres, A. R.; Lyra, W. S.; Andrade, S. I. E.; Andrade, R. A. N.; Silva, E. C.; Araújo, M. C. U.; Gaião, E. N.; Talanta 2011, 84, 501

32. Dai, X.; Xu, Y.; Zheng, J.; Ma, L.; Song, H.; Biosyst. Eng. 2020, 190, 157.

33. Sankar, K.; Lenisha, D.; Janaki, G.; Juliana, J.; Kumar, R. S.; Selvi, M. C.; Srinivasan, G.; Talanta 2020, 208, 120408.

34. Albizu, G.; Bordagaray, A.; Dávila, S.; Garcia-Arrona, R.; Ostra, M.; Vidal, M.; Microchem. J. 2020, 154, 104600.

35. Maleki, N.; Safavi, A.; Sedaghatpour, F.; Talanta 2004, 64, 830.

36. Zamora, L. L.; López, P. A.; Antón Fos, G. M.; Algarra, R. M.; Romero, A. M. M.; Calatayud, J. M.; Talanta 2011, 83, 1575.
37. Silva Lyra, W.; dos Santos, V. B.; Dionízio, A. G. G.; Martins, V. L.; Almeida, L. F.; Nóbrega Gaião, E.; Diniz, P. H. G. D.; Silva, E. C.; Araújo, M. C. U.; Talanta 2009, 77, 1584.

38. Chaplan, C. A.; Mitchell, H. T.; Martinez, A. W.; Anal. Methods 2014, 6, 1296.

39. Nitinaivinij, K.; Parnklang, T.; Thammacharoen, C.; Ekgasit, S.; Wongravee, K.; Anal. Methods 2014, 6, 9816.

40. Iqbal, Z.; Bjorklund, R. B.; Talanta 2011, 84, 1118.

41. Reile, C. G.; Rodríguez, M. S.; Fernandes, D. D. de S.; Gomes, A. de A.; Diniz, P. H. G. D.; Di Anibal, C. V.; Food Chem. 2020, 328, 127101.

42. Feiteira, F. N.; dos Reis, L. G. T.; Pacheco, W. F.; Cassella, R. J.; Microchem. J. 2015, 119, 44.

43. Catelani, T. A.; Bittar, D. B.; Pezza, L.; Pezza, H. R.; Talanta 2019, 196, 523.

44. Almeida, M. I. G. S.; Jayawardane, B. M.; Kolev, S. D.; McKelvie, I. D.; Talanta 2018, 177, 176.

45. Chanu, O. R.; Kapoor, A.; Karthik, V.; Mater. Today Proc. $2020,0$.

46. Luiz, V. H. M.; Lima, L. S.; Rossini, E. L.; Pezza, L.; Pezza, H. R.; Microchem. J. 2019, 147, 43.

47. Jia, B.; Wang, W.; Ni, X.; Lawrence, K. C.; Zhuang, H.; Yoon, S. C.; Gao, Z.; Chemom. Intell. Lab. Syst. 2020, 198, 103936.

48. Baiano, A.; J. Food Eng. 2017, 214, 10.

49. Capitan-Vallvey, L. F.; Nuria, L.; Martínez-olmos, A.; Erenas, M. M.; Palma, A. J.; Anal. Chim. Acta 2015, 899, 23.

50. Nuria Lopez-Ruiz, V. F. C.; Erenas, M. M.; Benito-Lopez, F.; Diamond, D.; Palma, A. J.; Capitan-Vallvey, L. F.; Anal. Chem. 2014, 86, 9554.

51. Feiteira, F. N. F. N.; dos Reis, L. G. T. L. G. T.; Pacheco, W. F. W. F.; Cassella, R. J. R. J.; Microchem. J. 2015, 119, 44.

52. Wyszecki, G.; Stiles, W. S.; Color Science: Concepts and Methods, Quantitative Data and Formulae, Wiley Classics Library, 2000.

53. Sonka, M.; Image Processing, Analysis, and Machine Vision, PWD Publishing, 2015.

54. Shishkin, Y.; Dmitrienko, S. G.; Medvedeva, O. M.; Badakova, S. A.; Pyatkova, L. N.; J. Anal. Chem. 2004, 59, 102.

55. Gomes, M. S.; Trevizan, L. C.; Nóbrega, J. A.; Kamogawa, M. Y.; Quim. Nova 2008, 31, 1577

56. Achterberg, E. P.; Holland, T. W.; Bowie, A. R.; Mantoura, R. F. C.; Worsfold, P. J.; Anal. Chim. Acta 2001, 442, 1.

57. Lin, M.; Hu, X.; Pan, D.; Han, H.; Talanta 2018, 188, 135.

58. Pacheco, W. F.; Farias, P. A. M.; Aucelio, R. Q.; Anal. Chim. Acta 2005, $549,67$.

59. Thompson, M.; Ellison, S. L. R.; Wood, R.; Pure Appl. Chem. 2002, 74 , 835.

60. Miller, J. C.; Miller, J. N.; Statistics for Analytical Chemistry, $3^{\text {rd }}$ ed., Prentice Hall: Upper Saddle River, 1993.

61. Hider, R.; Thalassemia Rep. 2014, 4, 19.

62. Turnquist, T. D.; Sandell, E. B.; Anal. Chim. Acta 1968, 42, 239.

63. Al-busafi, S. N.; Suliman, F. E. O.; Article, R.; Res. Rev. J. Chem. 2014, 3,1 .

64. Sandell, E. B.; Spindler, D. C.; J. Am. Chem. Soc. 1949, 71, 3806.

65. Prachayasittikul, V.; Prachayasittikul, S.; Ruchirawat, S.; Prachayasittikul, V.; Drug Des. Devel. Ther. 2013, 7, 1157.

66. Mouralian, C.; Buss, J. L.; Stranix, B.; Chin, J.; Ponka, P.; Biochem. Pharmacol. 2005, 71, 214.

67. Tewari, R.; Bachmann, G.; Hadacek, F.; Environ. Exp. Bot. 2015, 109 , 151.

68. Braun, T.; Navratil, J. D.; Farag, A. B.; Polyurethane Foam Sorbents in Separation Science, $1^{\text {st }}$ ed., CRC Press: Boca Raton, 1985.

69. Werbowesky, R.; Chow, A.; Talanta 1996, 43, 263.

70. Bratsch, S. G.; J. Phys. Chem. Ref. Data 1989, 18, 1.

71. Bard, A. J.; Parsons, R.; Jordan, J.; Standard Potentials in Aqueous Solutions, Marcel Dekker: New York, 1985.

72. Shahata, M. M.; Am. J. Chem. Appl. 2015, 2, 37. 
73. Deng, Y.; Water Res. 1997, 31, 1347.

74. Stefánsson, A.; Environ. Sci. Technol. 2007, 4, 6117.

75. Marguti, A. L.; Ferreira Filho, S. S.; Pivelli, R. P.; Rev. Eng. Sanitária e Ambient. 2008, 13, 395.

76. Firdaus, M. L.; Alwi, W.; Trinoveldi, F.; Rahayu, I.; Rahmidar, L.; Procedia Environ. Sci. 2014, 20, 298.
77. Choodum, A.; Sriprom, W.; Wongniramaikul, W.; Spectrochim. Acta, Part A 2019, 208, 40.

78. Santos, A. M. P.; Adriano, A.; Ferreira, S. L. C.; Talanta 2018, 194, 86.

79. Agência Nacional de Vigilância Sanitária (ANVISA); Guia para validação de métodos analíticos e bioanalíticos; Exatidão e Precisão. 